\title{
Aperçu des discours théoriques et critiques sur les desseins et engagements francophones : évolutions, dérives, ambiguiités
}

José Domingues de Almeida

\section{(2) OpenEdition \\ 12 Journals}

Édition électronique

URL : https://journals.openedition.org/dhfles/103

DOI : $10.4000 /$ dhfles. 103

ISSN : 2221-4038

Éditeur

Société Internationale pour l'Histoire du Français Langue Étrangère ou Seconde

Édition imprimée

Date de publication : 1 janvier 2008

Pagination : 63-76

ISSN : 0992-7654

\section{Référence électronique}

José Domingues de Almeida, «Aperçu des discours théoriques et critiques sur les desseins et engagements francophones : évolutions, dérives, ambiguïtés », Documents pour l'histoire du français langue étrangère ou seconde [En ligne], 40/41 | 2008, mis en ligne le 17 décembre 2010, consulté le 27 mai 2021. URL : http://journals.openedition.org/dhfles/103 ; DOI : https://doi.org/10.4000/dhfles.103

Ce document a été généré automatiquement le 27 mai 2021.

(c) SIHFLES 


\section{Aperçu des discours théoriques et critiques sur les desseins et engagements francophones : évolutions, dérives, ambiguïtés ${ }^{1}$}

José Domingues de Almeida

\section{Constat et attitude prospective}

1 Lorsque, en 1987, Jean-Marc Léger, acteur incontournable de la cause francophone, publie au lendemain du Sommet de Paris un essai sur le présent et l'avenir du français et de la francophonie, il le fait avec une rare clairvoyance, et surtout dans l'urgence, inspirée par la mauvaise passe dans laquelle il sent s'embourber la langue française comme langue de communication internationale. Cet essai signale, par le ton et le contenu, un point de bascule dans la compréhension même des enjeux de la francophonie comme présence de l'universel au monde, et pointe un horizon d'incertitudes, et d'idées à mettre en œuvre. Le journaliste se savait entre deux conceptions de la notion, du fait et de la machine francophone; entre deux discours: l'un spirituel et spontané ; l'autre, en devenir, et dès lors enclin à toutes les dérives et à tous les périls.

2 L'urgence d'abord. Nous sommes en 1987. Les signes précurseurs d'un effondrement international du rayonnement $\mathrm{du}$ français et de l'esprit francophone se précisent : « Il leur reste moins de deux décennies pour tenir le pari» (Léger $1987: 156)$; ou encore : «Force est de constater en effet qu'entre l'anglais et le français, la distance ne cesse de grandir. Si elle devait dépasser un certain seuil, une situation irréversible risquerait de se produire. Et cela va se passer dans les dix à vingt prochaines années » (idem : 168s). C'est désormais chose faite! Léger déplore aussi la marginalisation du FLE (français langue étrangère) : «Or, cette évolution déjà fortement avancée risque de se précipiter dans les deux ou trois décennies qui viennent » (idem : 172). Et Léger de conclure, pour 
ne pas dire de nous conjurer d'agir : « Il faudra faire vite sans pour autant chercher à imposer un modèle, une démarche, des structures contraignantes, une déclaration ou une charte quelconque de la francophonie " (idem : 179). La clairvoyance, ensuite. Tant il est vrai que le responsable québécois s'est avéré d'un rare prophétisme quand il s'est agi d'entrevoir, de prévoir l'avenir des francophonies dans leur projections internationales et géopolitiques, c'est-à-dire les seules qui comptent en dehors de toute logique folklorique ou exotique.

1987, Léger alertait contre les dangers qui guettaient déjà, mais n'osaient pas encore dire leurs noms :

En ce qui concerne le français, dans le monde d'aujourd'hui, (où il garde ses chances, si les francophones veulent et savent les exploiter), il apparait comme le grand recours contre un néo-impérialisme technocratique et contre la déshumanisation, sous réserve qu'il sache répondre aux deux vocations nouvelles que lui propose l'époque : être la langue de la coopération et dès lors du dialogue des cultures, être une langue porteuse de progrès et de modernité. » (idem : 174) ${ }^{2}$

4 Et Léger de cerner les défis futurs avec une impressionnante lucidité : «Le combat majeur de demain sera celui de l'identité. » (idem : 175)

5 Vingt ans plus tard, ces prévisions résonnent avec une douloureuse acuité. Que s'est-il passé entre-temps? Un emballement de l'Histoire peu favorable aux vœux pieux des discours francophones dont Jean-Marc Léger se méfiait, la chute du Mur, le changement de la géographie européenne et des grandes aires géolinguistiques; un souffle libéral sur un Occident homogénéisé par l'économie et l'anglais, Internet et la mondialisation; le dégagement d'autres aires émergentes sur fond géopolitique hérité de la guerre froide faisant ombrage à la francophonie comme garante de la diversité culturelle: l'espagnol, l'arabe ou le chinois. Le français perd du terrain sur tous les tableaux, notamment sur la scène diplomatique, le tout sur un fond multiculturel des plus chancelants.

6 Léger avait été le témoin d'une lente et libre construction francophone dans la foulée des décolonisations. Des initiatives spontanées de visionnaires (dont les Pères fondateurs), des universitaires, des journalistes, des hommes de culture, certains hommes politiques aussi, avaient mis sur pied une idée porteuse d'avenir, une communauté d'affinités fondée sur l'universalité de la langue française.

7 C'est justement ce discours et cette posture qui prévalurent au lendemain de la geste décolonisatrice, notamment à Niamey en 1969, qui enfanta une véritable koinè francophone, humaniste et spirituelle, nourrie, entre autres, par les sentiments de la négritude: "Il y a d'ailleurs [...] une sorte de filiation spirituelle entre négritude et francophonie. » (idem : 58) (apud idem : 104)

8 À ce stade, le discours francophone fait converger, par un élan spirituel, « la conviction du caractère universel du français et de son aptitude particulière à porter, à exprimer, à accueillir les diverses cultures » (idem : 59), avec la mise sur pied d'une première organisation de coopération (l'ACCT) que les sommets francophones et l'OIF relayeront plus tard et dans un tout autre contexte. Il devient très vite clair que, dans l'esprit et les faits, la francophonie ne sera jamais une sorte de Commonwealth, davantage " [...] tourné vers l'économie $»^{3}$ (Wolton $2006: 45$ ).

9 Après l'esprit de «l'émergence du domaine et du monde francophones » qu'il a vécu, et le Sommet de Paris de 1986, Jean-Marc Léger exprime toutes les craintes de voir cette 
aventure francophone sombrer dans l'impasse. Tous les espoirs sont permis. L'entreprise a tout pour aboutir :

La communauté est en voie de s'édifier autour de trois idées majeures : la maîtrise en commun des nouvelles technologies, particulièrement en matière de communication et d'informa-tion ; le salut des identités nationales, c'est-à-dire des cultures de tous les peuples concernés; une conception nouvelle de la coopération qui à la notion d'aide ou d'assistance substitue celle de partage dans son sens le plus large. (Léger $1987: 153$ )

Et, cependant, le ton est foncièrement pessimiste, un rien pouvant tout faire échouer : «Il est significatif que la langue française soit le lieu et le moyen de cette initiative historique, puisque son passé et sa situation actuelle dans le monde en font à la fois la plus illustre, la plus visible mais aussi la plus menacée des autres, une forme de vigie et peut-être de recours » (ibidem). Somme du contexte extrinsèque où l'américanisation à outrance force la « clochardisation des cultures » (idem : 154), la disparition de langues et de la diversité culturelle par une uniformatisation anglo-saxonne, et d'une démission intrinsèque côté francophone, surtout français, à l'égard de la cause des Pères fondateurs.

\section{Apories des discours}

11 Un temps, pendant le répit qui précéda la chute du Mur, le discours francophone fut tenté d'épouser le "non-alignement» (idem: 160). Le français était une langue «nonalignée » du fait de sa diffusion mondiale et du positionnement idéologique et politique sur l'échiquier stratégique international des nouveaux états décolonisés.

La fin des alignements idéologiques et la mondialisation devaient dicter la fin de cette dernière chance de récupération, et mettre à nu les handicaps intrinsèques liés à l'usage de cette langue, et qui influent sur l'affirmation internationale de la francophonie et sur la substance même des «études francophones ". D'une part, il y a la centralité exclusive et excessive de la France (et de Paris) dans le pôle francophone comme concentration des instances de légitimité et théâtre de l'hypostase de la langue comme affaire d'État (idem: 161$)^{4}$, et le statut que cela impose aux périphéries. Car, qu'on le veuille ou non, et contrairement à tous les autres ensembles linguistiques et culturels (Commonwealth, hispanidad, lusophonie ou germanophonie), la francophonie ne connait pas le pluri-centrisme éditorial, légitimant et, dès lors, référentiel. Elle n'a pas non plus engendré un contrepoids géosymbolique capable de contrecarrer cet état des choses. De ce fait, Paris concentre les instances de légitimation qui confèrent la (re)connaissance et la valeur aux œuvres et aux produits culturels, qu'ils soient français ou ... francophones (Klinkenberg 1984 : 31).

La France se fait attendre sur la scène internationale. La déprime et le déclinisme hexagonaux, forts remarqués, parfois même regrettés à l'étranger (Anderson, 2005), après le verbe gaullien, et ce malgré de remarquables réussites technologiques et quelques résultats consistants sur le dossier très hexagonal, relayé par l'organigramme francophone, de l'«exception culturelle", affectent sérieusement le rayonnement francophone ; et il n'est pas dit que la France sarkoziste puisse inverser le cours !

La centralité exclusive de la France contraint l'Hexagone à mener le jeu de l'inventivité et de la puissance dans tous les domaines culturel, scientifique ou technologique (Léger 
1987 : 165) de telle sorte que c'est l'image de la France qui continue de détourner le rayonnement géopolitique, géosymbolique des francophonies.

D'autre part, il y a la question cruciale de la langue. Or, la langue française, et ce contrairement aux autres grandes langues européennes de communication internationale, n'a pas développé un cheminement d'inclusion de la diversité, ne s'est pas habituée à dire des histoires autres que la saga républicaine française, et ce en dépit de son passé colonial et de sa conjoncture multiculturelle; ce qui induit un sentiment de dépossession linguistique et discursive dans les périphéries francophones. Le processus historique français de centralisation et d'hypostase prescriptive du beau langage, surtout après la centralisation monarchique, l'Académie française, la Révolution, la République, l'école laïque et l'évincement des réalités dialectales, a abouti à deux conséquences de taille pour le rayonnement actuel du français, et explique, pour une large part, la situation présente caractérisée par l'impasse.

Autrement dit, la France est devenue, sans trop s'en rendre compte, une nation linguistique, et, partant, une nation littéraire au sens que Priscilla Parkhurst Ferguson donne à cette expression : « le champ littéraire français aime à se signaler à l'attention, comme pour transmettre à la société son capital d'idées et d'idéaux, par toutes sortes de représentations emblématiques ou symboliques » (Ferguson 1991 : 24). De ce fait, le français subit les désagréments de sa connotation élitiste passée, comme le suggère Claude Hagège :

Le français continue d'apparaître comme une langue plus fortement que toute autre liée à une littérature, à une pensée critique, à une culture. Le français ne semble jamais être devenu ce qu'est aujourd'hui l'anglais [mais on pourrait invoquer ici d'autres langues à vocation internationale] une pure langue véhiculaire débarrassée de toute référence à un enracinement historique et à une forme de civilisation. (Hagège $2006: 175)^{5}$

D'une part, ce processus a terriblement compromis les chances d'épanouissement de la langue instrumentale et existentielle au profit d'une conception monumentale et essentialiste du beau langage, pour citer Jean-Marie Klinkenberg (apud Renard 2003 : 184).

Mais, d'autre part, les marges périphériques francophones ne participent pas, qu'elles le veuillent ou non, à cette aventure destinale et nationale de la langue, à moins qu'elles ne décident tout simplement de dénier et de forclore une réalité propre, une Histoire autre qui a à se dire en français; ce qui fut trop souvent le cas au Québec, en Suisse, en Belgique ou aux Antilles avant les révolutions mentales et littéraires que l'on sait, de la belgitude à Édouard Glissant ; du renouveau québécois à la suissitude. Dès lors, «dénationaliser la langue» (Jacob 2006), c'est-à-dire, quelque part, la "déterritorialiser ", devient une tâche, voire une revendication consensuelle dans les cahiers de doléances francophones. Et enfin, il y a le rapport complexé et problématique à l'Histoire côté francophone. Raison pour laquelle aux périphéries ne peuvent s'appliquer les contraintes et la conception historiale de la France, Monarchie et République confondues, et a fortiori, la conception romantique de l'Histoire nationale telle que le XIX ${ }^{e}$ siècle l'a encensée, notamment par la littérature.

19 L'imposition de pareille vision fait apparaître ces régions comme des no man's lands historiques, comme des aires frappées d'anhistoricité ou d'amnésie. Elle débouche forcément sur l'emprunt refoulé d'une histoire qui n'est pas la leur. Ce fut le cas pour la Belgique, ou même pour la Suisse ou le Québec, auxquels on a imposé une copie non 
conforme à l'Histoire propre à ces périphéries. Ce travail idéologique, castrateur, a souvent dénaturé le passé subsidiaire, fédéral ou baroque de ces pays pluriels, et qui se savent tels depuis le Moyen-Âge, c'est-à-dire bien avant la saga républicaine française en tout cas. Cette imposition a, par ailleurs, inculqué une conception de francité coloniale qui a débouché sur une véritable négation, voire falsification ou révision implicites de l'Histoire; c'est-à-dire la mise sous silence des histoires périphériques dérogeant au canon historial français. Enseigner l'Histoire en contexte multiculturel, c'est-à-dire en tenant compte des nouvelles réalités sociétales françaises, s'avère un défi majeur eu égard au passé colonial français, à ses retombées en flux migratoires et en convivialités interculturelles et, on oublie trop souvent d'y faire allusion, à la politique francophone de la République « une et indivisible ».

\section{Équivoques et dérives}

20 Qui plus est, aux dires de Pascal Blanchard, dans l'excellent recueil de témoignages sur l'état actuel de la France: «La 'fracture coloniale' désigne d'abord un rapport à l'histoire et à la mémoire. Avec le Japon, sans doute, la France est la seule nation au monde qui n'a pas encore pleinement intégré dans sa mémoire nationale - et collective - ce 'temps colonial'. Cela constitue une sorte de fracture ou de césure dans son rapport à l'histoire » (Finkielkraut $2007: 43$ ). Conséquence majeure entre autres de ce fait, selon le même auteur: "la manière dont nous pensons la francophonie» (idem: 44). De même, et dans une perspective tout aussi cohérente, Jean-Pierre Obin s'interroge sur la possibilité de décliner cette histoire unique et linéaire à l'heure des intégrations difficiles ou improbables: «[...] est-ce que l'enseignement de l'histoire peut être la simple juxtaposition d'histoires particulières, celles des populations qui ont progressivement constitué la nation française? Ou bien existe-t-il une histoire de France qui est celle d'une unité politique qui elle aussi s'est constituée progressivement?» (idem: 55). D'autant plus que, à l'instar de l'individu, « une communauté n'a d'identité qu'à travers les histoires qu'elle a vécues ou qu'elle se raconte » (idem : 232). D'où la question cruciale pour notre propos : quelles histoires les périphéries francophones se racontent-elles qu'elles aient réellement vécues?

21 Ces écueils et apories n'épuisent pas, loin s'en faut, les raisons de l'impasse où se trouve l'aire géolinguistique francophone sur le plan international. Dans un contexte mondial entièrement nouveau, de nouveaux discours s'emparent du projet francophone et le mettent au goût du jour. Ainsi, dans bien des essais parus ces dernières années, la Francophonie, faute de dessein géopolitique global consistant, vient se greffer et porter main forte aux interrogations et aux soucis thématiques du moment. C'est précisément le cas du discours antilibéral assumé ou emprunté par toute une mouvance francofrancophone sur la défensive, qui assigne à la géopolitique francophone la sale besogne de servir de (dernier) rempart à une mondialisation sauvage, à la globalisation anglosaxonne. Cette position consensuelle dans le discours francophone actuel mérite que l'on s'attarde un temps soit peu sur la portée des arguments mis en exergue.

Chez les essayistes en question, de l'altermondialisme de Porto Alegre et de M. Bové (qui parle anglais à ses heures !) à la francophonie géopolitique, il n'y a qu'un pas qu'ils franchissent allégrement au nom d'une France frileuse, intimidée, gauchiste ou en marge des processus d'échange en marche sur la planète. Les expressions sont on ne peut plus claires : «Or il existe une alternative [à la mondialisation], nous l'avons sous 
les yeux mais ne la voyons pas. La Francophonie, comme les autres aires culturelles, en est un exemple concret. » (Wolton $2006: 19$ )

Claude Hagège tient à peu de choses près le même langage :

Cela met la francophonie en mesure de donner un contenu réel à la mondialisation, au-delà des considérations de pur profit dont celle-ci est le prétexte, utilisé par les grandes firmes multinationales. Ou, plutôt, cela offre la possibilité de proposer, face au rêve américain, dont le type actuel de mondialisation est la forme contemporaine, un autre rêve, altermondialiste et surtout humaniste ${ }^{6}$. (Hagège, $2006: 154)$

Le "grand large" auquel se réfère constamment Serge Arnaud comme possible extension de la France en ces temps globalisés coïncide avec un espace mondialisé ; qui refuse la globalization de la superpuissance américaine, et ce pour conclure que : «la Francophonie peut rendre 'un autre monde possible'« (Arnaud et al. 2005: 18); ou encore, très explicitement : «La Francophonie est une autre mondialisation.» (idem : 53)

25 C'est dire combien, par le discours francophone actuel, la francophonie se confond, très à gauche et de façon tout à fait idéologique, quoique subtile, avec l'espoir humaniste d'une mondialisation autre qui puisse se dresser en rempart contre la fascination libérale anglo-saxonne. Hagège va plus loin. Selon lui, il y a même lieu d'interchanger le discours libéral et langue anglaise. Cette hantise antilibérale nuit gravement à la projection internationale du français. Elle prive le français d'un accès immédiat et sans mauvaise conscience aux domaines de la finance et de l'économie. C'est même oublier que le (néo)libéralisme n'éveille pas partout les mêmes soupçons. Des histoires francophones décalées, certes, par rapport à l'Histoire de France, comme celles de la Belgique ou de la Suisse, se sont bâties sur des assises libérales.

Par ailleurs, il serait pour le moins intellectuellement déconcertant que la francophonie ne fût envisageable ou interrogeable que selon un discours gauchiste, ou plus ou moins altermondialiste. Dominique Wolton, dans un accès de bon sens, ne conclut-il pas $\mathrm{qu}^{\prime}$ « elle [la francophonie] doit pouvoir conjuguer logique intergouvernementale et logique altermondialiste ", et que la langue française " [...] doit également s'accrocher aux nouvelles réalités de la mondialisation, être aussi une langue de l'économie, du commerce et de la société » (Wolton 2006 : 188). D'autant plus que, comme prévient Raymond Renard, il n'est pas de langue spécifique de l'économie (Renard 2003: 52), si ce n'est en préjugé.

Même logique partielle, voire partiale, quand il s'agit de faire coller à tout prix la Francophonie à la mouvance multiculti (multiculturalisme) qui déferle sur les campus européens, souvent en provenance des États-Unis d'Amérique. Certes, nos sociétés sont devenues, à des degrés divers, "multiculturelles", notamment la société française ; une caractéristique impliquant une complexification et un enrichissement de la notion d'Histoire nationale dans la foulée de la décolonisation; celle-là même qui, nous le disions, pose problème à un juste rééquilibrage des espaces francophones, centre et périphéries.

28 François Durpaire, dans un (r)apport didactique pertinent à la question multiculturelle dans ses implications historiographiques, se fait l'écho de ce malaise :

L'histoire de France connaît depuis lors une difficulté à inclure en son sein la dualité, la contradiction, la dialectique. Il semble que la France ne puisse pas accepter la totalité de son passé et soit destinée à privilégier une partie d'elle- 
même. L'histoire reste ainsi partielle, comme s'il ne pouvait y avoir qu'une France et une seule tout au long de son histoire. (2002:28) langues menacées, selon les circonstances, et l'anglais n'atteint pas également toutes les aires géoculturelles ou linguistiques. Il ne menace ni l'espagnol, ni le chinois, ni même l'arabe. Ces langues en viennent à être menaçantes, même pour l'anglais, comme c'est le cas aux États-Unis; ce qu'attestent les lois d'imposition de l'anglais dans certains États.

Or, le français apparaît de plus en plus aux yeux des allophones comme une langue décadente, liée à une culture en déclin; ce dont les francophones de souche, et les Français en tête, ne se rendent pas toujours compte ${ }^{7}$. Et l'espagnol, par exemple, se charge, sans trop se l'avouer, c'est-à-dire sans esprit de mission, de la tâche difficile et improbable de contrecarrer l'américanisation par l'apport d'une voix/voie autre ; une sale besogne que la Francophonie se réserve(ait) (Hagège 2006) ; ce qui risque de vider son programme, sa raison d'être sur l'échiquier géoculturel. Or, chez bien des théoriciens et essayistes du fait francophone de ces derniers temps, le rôle assigné à la Francophonie est d'apparaître en experte en diversité et en dialogue des cultures et des langues ; ce qui n'est pas faux du reste, mais n'en pose pas moins problème eu égard à l'image souvent arrogante ou imbriquée dans l'idée d' « exception » que l'on se fait de la France en dehors même des espaces francophones.

D'ailleurs, il n'est pas dit que ce vote à l'Unesco soit une victoire de, voire pour la francophonie, comme le suggère Dominique Wolton (2006 : 9), qui rappelle, ou avoue, au passage que : «[...] la France, pays jacobin, centralisateur, se méfie de tout ce qui s'appelle diversité » (idem : 23). Mais l'idée fait son chemin, dans le discours, qu'il revient au français, en se sauvant lui-même, de sauver la communication multiculturelle planétaire. 
effronterie, qui a des relents de faiblesse mal gérée, risque d'agacer les interlocuteurs du débat interculturel. D'autant plus que ce dessein en cache un autre, qui n'ose pas dire son nom : procurer à la France une dernière aire de rayonnement en tant que puissance moyenne déclinante ; retour voilé de l'excessive centralité française dans les francophonies. Dominique Wolton laisse deviner ce dessein :

La situation n'est donc pas du tout brillante pour la langue française. Il y a même beaucoup à faire, et vite, si l'on veut conserver au français un statut de langue internationale. Ce que n'ont pas compris les 'réalistes'? Seule la francophonie donnera demain un statut mondial à la France... (idem : 18)

\section{Espoirs : gérer le réalisme et le volontarisme}

Il ressort des considérations que nous venons de tenir que nous sommes, côté francophone, devant une situation certes prestigieuse mais relativement bloquée. En fait, l'émergence du monde francophone, de par sa particularité et complexité, entraîne avec elle la formulation de discours d'escorte changeant en fonction des aléas géolinguistiques du français. Faut-il regretter ces tergiversations discursives au goût du jour? Le fait que les discours n'épousent que la partie au lieu du tout? La défensive au lieu du projet qui passe avant tout par le rayonnement de sa raison d'être : le français? Une définition en creux, en somme, au lieu d'un plein proactif? Elles trahissent en tous cas l'incapacité francophone d'aujourd'hui à apparaître comme un tout évident et consistant, allant de soi, et ayant une présence au monde indiscutable, indiscutée; ce qu'avait été le dessein des fondateurs quand ils sublimaient l'universalité du français et sa capacité à accueillir les cultures. Il s'agit, dès lors, de faire sortir la francophonie, ou plutôt les francophonies, du cadre national, et de voir quelque chose, autre chose qu'une certaine coloration exotique de la belle langue française, qu'une ONG, bonne à tout faire.

Il s'agit pour la France, moteur historique obligé du français, de remettre en marche sa machine contestataire du social et de l'établi, et de procurer au monde des raisons et des mots en plus des technologies et des performances. À en croire certaines voix, c'est sur ce terrain qu'on l'attend (Anderson 2005 : 94-97). Comme le signalait Jean-Marc Léger, la francophonie ne peut pas être une construction artificielle. Elle ressortit aussi bien à l'initiative spontanée qu'étatique. Cet espace ouvert prend chez Dominique Wolton les traits d'une francosphère, prolongement du désir de France et du français sur la scène internationale (2006: 73). Il assume chez Serge Arnaud l'aspect d'un grand large géopolitique touchant à toutes les présences françaises dans le monde, notamment dans les liens étroits qu'elles ont tissés avec d'autres cultures et d'autres langues (Arnaud et al. 2005 : 57-61). M. Sarkozy, qui ne s'est pas encore vraiment investi dans la cause francophone, parle d'un dialogue autour de la Méditerranée. A-t-il l'intention de le tenir en français et au nom des francophonies? Ce serait une excellente occasion d'ajuster le discours et la réalité, de suppléer à un creux discursif. 


\section{BIBLIOGRAPHIE}

ANDERSON, Perry (2005). La pensée tiède. Un regard critique sur la culture française. Paris, Seuil. ARNAUD, Serge et al. (2005). Les défis de la francophonie. Pour une mondialisation humaniste. Paris, Alpharès.

BARREAU, Jean-Claude (1997). La France va-t-elle disparaître ?. Paris, Grasset.

COÛTEAUX, Paul-Marie (2006). Être et parler français. Paris, Perrin.

DURPAIRE, François (2002). Enseignement de l'histoire et diversité culturelle. « Nos ancêtres ne sont pas les Gaulois ». Paris, Hachette.

FERGUSON, Priscilla Parkurst (1991). La France, nation littéraire. Bruxelles, Labor.

FINKIELKRAUT, Alain (2007). Qu'est-ce que la France ?. Paris, Stock/Panama.

HAGÈGE, Claude (2006). Combat pour le français. Au nom de la diversité des langues et des cultures. Paris, Odile Jacob.

JACOB, Didier (2006). « Les écrivains francophones en colère. 'Nous accusons la France'«, Le Nouvel Observateur, 16-22 mars.

KLINKENBERG, Jean-Marie (1984). « Pour une étude de l'institution littéraire en Belgique francophone », in Écriture française et identifications culturelles en Belgique. Louvain-la-Neuve, CIACO.

LÉGER, Jean-Marc (1987). La Francophonie : grand dessein, grande ambiguïté. Paris, Nathan.

RENARD, Raymond (2003). Une éthique pour la francophonie. Questions de politique linguistique. Paris/Mons, Didier/CIAPA.

WOLTON, Dominique (2006). Demain la francophonie. Paris, Flammarion.

\section{NOTES}

1. Cette communication a été élaborée dans le cadre du projet «Inter-identidades » de l'Institut de Literatura Comparada Margarida Losa de la faculté des Lettres de l'université de Porto, une I\&D subventionnée par la Fundação para a Ciência e a Tecnologia, intégrée dans le «Programa Operacional Ciência, Tecnologia e Inovação (POCTI) », Quadro de Apoio III (POCTI-SFA-18-500).

2. C'est nous qui soulignons.

3. Ce que Jean-Marc Léger appelait « une comparaison trompeuse » (Léger 1987 : 65-71).

4. Voir l'argument inverse chez Jean-Claude Barreau (1997: 173).

5. Paul-Marie Coûteaux relaie cette intuition quand il s'interroge sur le fait que «Peut-être la France est-elle une nation trop littéraire pour être en paix avec sa langue. » (Coûteaux 2006: 73)

6. C'est nous qui soulignons.

7. C'est le cas au Portugal notamment où l'espagnol a été introduit comme deuxième langue étrangère du secondaire, bénéficiant d'une large adhésion dont le français paie les frais. 


\section{RÉSUMÉS}

Le projet francophone ne s'est jamais vraiment présenté de façon cohérente depuis les indépendances. Il a connu des moments clés, des points de bascule décisifs, mais a également fait l'objet d'un essayisme prospectif. Mais force est de reconnaître que cette pensée critique signale quelques évolutions, voire quelques dérives discursives et argumentatives en ce qui concerne l'image de la France en France et dans les Francophonies. L'auteur suggère un aperçu de ces balises depuis les indépendances jusqu'aux récents essayistes des études francophones D. Wolton, J.-M. Léger, Cl. Hagège, S. Arnaud ou R. Renard.

The francophone project has never been completely coherent since the independences. It has known key moments, decisive turning points, but has also been followed by a prospective essayism. But, one has to recognize that this critical thought builds some evolutions or even discursive and argumentative abuses as far as the image of France in France and in French speaking countries is concerned. The author suggests a survey of these marks from the independences to the Francophone studies lately in essayists like D. Wolton, J.-M. Léger, Cl. Hagège, S. Arnaud or R. Renard.

\section{INDEX}

Mots-clés : francophonie, discours, ambiguïtés, français, culture

Keywords : Francophonie, discourses, ambiguities, french, culture

\section{AUTEUR}

\section{JOSÉ DOMINGUES DE ALMEIDA}

Université de Porto

Instituto de Literatura Comparada Margarida Losa

Portugal

jalmeida@letras.up.pt 